\title{
Oxidative stress, plasma/salivary antioxidant status detection and health risk factors
}

\author{
Vadalà Maria', Palmieri Beniamino², Malagoli Andrea ${ }^{3}$, Laurino Carmen ${ }^{4}$ \\ ${ }^{1,4}$ Research, ${ }^{2}$ Professor, Department of General Surgery and Surgical Specialties, ${ }^{3}$ Researcher, Department of Medical \\ and Surgical Sciences for Children \& Adults, University of Modena and Reggio Emilia, Modena, Italy
}

Background: Oxidative stress is involved in the pathophysiology of several diseases such as cardiovascular disorders, cancer, neurodegeneration. Aims and Objectives: Our study evaluated the oxidant/antioxidant status on a cohort of healthy patients matched with some independent variables as a basic individual redox balance monitor on a disease-prevention perspective. Materials and Methods: The anecdotic, retrospective and observational study included 200 apparently healthy volunteers after formal informed consent release whose personal history and physical examination had been recorded specifically on the following items: age, previous diseases, sport activities, smoking habit, balanced/unbalanced nutrition, current absence/presence of inflammatory processes, oral health hygiene, administration of oral contraceptives or hormone replacement therapy in postmenopausal women. No drug treatment was admitted in the recruited patients, up to 6 months before the evaluation. The laboratory instruments used were Point of care FRAS 4 Evolvo (H\&D, Parma, Italy) measuring the oxidative stress in plasma samples and antioxidant capacity in plasma and saliva samples. Results: Two-hundred patients were recruited. Statistically relevant differences were observed in oxidative stress-related variables, namely a significant relationship between plasma oxidative stress level and female gender $(p<0.01)$, between saliva antioxidant level and age $(p=0.01)$, between plasma antioxidant level and unbalanced diet $(p<0.01)$, between plasma oxidative stress level and inflammation in the oral cavity $(p=0.04)$, and between saliva antioxidant level and inflammation in the oral cavity $(p<0.01)$. Conclusions: A relationship between oxidative/antioxidant status and health risk factors has been outlined in our study; the achieved data are quite helpful, in the clinical practice, providing additional information on individual general health conditions, putatively related to prevention diagnosis, prognosis, and treatment effectiveness in some specific diseases.

Key words: Antioxidant, Factor, Oxidative, Plasma, Stress, Risk, Test, Saliva

\section{INTRODUCTION}

Reactive oxygen species (ROS) are produced by molecular oxygen as a result of physiological cell metabolism and are subdivided into 2 groups:

1) Free radicals, molecules that have one or more unpaired electrons in their valence orbital [e.g. superoxide anion $\left(\mathrm{O}_{2}^{-}\right)$, hydroxyl radical $(\mathrm{OH})$ and nitric oxide $(\mathrm{NO})]$

2) Non radical oxygen derivatives [e.g. hydrogen peroxide $\left(\mathrm{H}_{2} \mathrm{O}_{2}\right)$, hypochlorous acid $\left.(\mathrm{HOCl})\right] .{ }^{1}$

\section{Access this article online}

Website:

http://nepjol.info/index.php/AJMS

DOI: 10.3126/ajms.v8i1.15134

E-ISSN: 2091-0576

P-ISSN: 2467-9100 
molecules including aminoacids (tyrosine, tryptophan, histidine and cysteine) lipids, proteins and DNA. ${ }^{4}$

These cytotoxic effects are largely responsible of ageing processes, diabetes, cardiovascular disorders, neurodegenerative diseases, and cancer.

\section{Oxidative stress and health risk factors}

The main health risk factors involved in the oxidative stress are gender, age, tobacco smoking, unbalanced diet, inflammatory processes (e.g. gingivitis, periodontitis), hormonal treatment for women.

\section{Gender}

Vignon-Zellweger and coworkers ${ }^{5}$ supposed that the sexual dimorphisms in mice cardiac dysfunction could be due to a gender difference in the expression pattern of endothelin-1 gene and endothelial nitric oxide synthase (eNOS) proteins involved in cardiovascular system, enhanced by sex hormones such as estrogen and 17-beta-estradiol at the transcriptional and post-translational level. ${ }^{6,7}$ Estrogen upregulates eNOS and activates a phosphatidylinositol 3-kinase (PI3K) signaling, leading to phosphorylation and activation of eNOS and to increase of $\mathrm{NO}$ signaling in target tissue. ${ }^{3}$ A clinical trial also outlined a potential link between high oxidative stress level and progression of autoimmune disorder, thrombocytopenia, in the women when compared with men. ${ }^{8}$ The oxidative stress upregulates cortisol by the adrenal gland which increases platelet production as a stress response. ${ }^{9}$

However, gender-specific differences might play a significant role in the pathophysiology of diseases and should be taken into consideration in future research.

\section{Age}

In the ageing process, the oxidative stress, generated throughout the lifespan, can lead to a vicious cycle in which damaged mitochondria produce increased amounts of ROS, leading to progressively worsening DNA damage. ${ }^{10}$ Indeed, the elderly subjects show an increased oxidative stress and decreased antioxidant levels. ${ }^{11}$

\section{Tobacco smoking}

Cigarette smoke has a gaseous and tar phase, with inorganic and organic free radicals, such as ROS, epoxides, peroxides, $\mathrm{NO}$, nitrogen dioxide, peroxynitrates and peroxynitrite. ${ }^{12}$ More than 4700 chemicals with a high concentration $\left(10^{15}\right.$ free radicals, including $\mathrm{O}_{2}{ }^{-}$and $\mathrm{NO}$ ) have been detected. ${ }^{13}$ They deplete tissues of ascorbic acid and protein sulfhydryl groups, causing the oxidation of DNA, lipids and proteins. The majority of healthy smokers has high levels of circulating F2-isoprostanes and urinary excretion rates of oxidized DNA products, confirming the oxidative stress. ${ }^{14}$

\section{Unbalanced diet}

A balanced diet provides macronutrients $(50-60 \%$ of carbohydrates, $20-30 \%$ fats, $10-15 \%$ proteins) and micronutrients [minerals, antioxidants (Vitamin C, E, $\beta$-carotene, riboflavin) and phytochemicals (polyphenols, flavonoids)] through an intake of basic four food groups: 1) Cereals, millets and pulses, 2) Vegetables and fruits, 3) Milk and milk products, egg, meat and fish, 4) Oils, fats, nuts and oilseed. ${ }^{15}$ The required amount of nutrients varies with age, gender, physiological status and physical activity of the individual. An insufficient amount or excess of the recommended food groups is defined unbalanced diet, such as a diet high in fat, particularly saturated fat, and low in carbohydrates, fruit, and vegetables. The nutritional background is probably involved in chronic inflammatory, autoimmune and vascular diseases, and also cancer.

\section{Inflammatory processes}

The unbalanced oxidant/antioxidant status is responsible through extracellular matrix (ECM) damage and accelerated apoptosis of worsening of diabetes, myocardial infarction, rheumatoid arthritis, sepsis, cancer, and Alzheimer's disease. ${ }^{16}$ High level of oxidative stress may in fact degrade ECM and necrotic cells, release intracellular and extracellular molecules, defined pathogen-associated molecular patterns (PAMPs), which act as 'alarmisms', generating the so-called oxidation-specific epitopes (such as oxidized phospholipids, oxidized cholesteryl esters and oxidized low-density lipoporoteins), that trigger inflammatory cascades through binding to multiple pattern recognition receptors (PRRs). ${ }^{17}$

\section{Use of oral contraceptives or estrogen-progestin treatment for female patients}

The oral contraceptives (different types of synthetic estrogens and progestins) administration is first introduced in the $1957 \mathrm{~s}$, is widely used by millions of women worldwide. ${ }^{18-20}$ In postmenopausal women, hormone replacement therapy is based on different hormonal mix [including estrogens (estradiol and conjugated estrogens), natural progesterone or synthetic progestins] to replace the failing ovarian secretion. ${ }^{19}$

Experimental and clinical studies on the oral contraceptives administration and oxidative stress showed a significant increase in lipid peroxidation, responsible for increased platelet aggregation in rats ${ }^{21-24}$ and also a significant decrease in plasma antioxidant $\beta$-carotene level in women above the age of 35 years. ${ }^{25}$ However, these side effects of the use of oral contraceptives might be mediated by ROS overproduction.

The oral contraceptives role in cancerogensis and cancer death is not definite. It is supposed that estrogen binds to 
cell receptors, which upregulate hormone responsive genes and then promote DNA synthesis and cell proliferation, that in genetically mutated nuclei induce malignant tumors. ${ }^{26}$

\section{Searching criteria}

The aim of this study has been to evaluate on an apparent healthy population the oxidant/antioxidant balance with specific comparison of plasma versus salivary antioxidant pool and to correlate a number of independent variables to the oxidative stress in a disease preventing perspective.

\section{MATERIALS AND METHODS}

The anecdotic and retrospective observational study recruited a cohort of 200 apparently healthy volunteers (100 men and 100 women, 10-94 years old), referred to the "Second Opinion Medical Network" that is a consultation referral web and Medical Office System involving a wide panel of specialists to which patients affected by different diseases not adequately satisfied in terms of diagnosis and treatment can apply for a clinical update. ${ }^{27}$ Most of the patients, nowadays in fact, often wonder around the medical Web-sites, looking for proper answers to their health problems, but this screening is often excessive, compulsive, and sometimes obsessive, leading to the "Web Babel Syndrome (this term expresses a doctor-patient communication gap that especially when affected by multiple synchronous pathologies, feeds back heterogeneous and misleading informations and prescriptions with the risk of a confusionary state. ${ }^{28,29}$ To solve this problem, the "Second Opinion Network" represents an useful decision-support tool not only in order to achieve a re-evaluation of the patient's case with a consequent optimization of treatment and prognosis, but also to avoid un-necessary investigational procedures, undue unhelpful and expensive medical and surgical treatments.

The selected patients were informed, via individual interview, and Informed Consent previously approved by the Local Institutional Review Board under the Helsinki Declaration. Contemporarily, background information regarding detailed personal history and physical examination was recorded specifically on the following items: age, previous diseases, current absence of inflammatory processes, oral health hygiene, smoking habit, sport activities, balanced/unbalanced nutrition enclosing satisfying amount of basic four food groups, in particular of antioxidants sources (fruits, vegetables) and, juice, tea, wine, coffee consumption; and administration of oral contraceptives or hormone replacement therapy in postmenopausal women. No current, previous (up to 6 months), drug treatment was admitted in the recruited patients. In the smoking group, 46 patients $(23 \%)$ were intense smokers (smoking more than 10 cigarettes per day since 5 years), 27 patients $(14 \%)$ were moderate smokers (smoking less than 5-10 cigarettes per day since 5 years), while 127 (64\%) were non smokers (who had smoked less than one cigarette per day for $<1$ year in their life). One hundred twenty patients $(60 \%)$ had an unbalanced diet and 113 subjects $(56.5 \%)$ registered an inflammatory process in the oral cavity (e.g. gingivitis, periodontitis). Among women, 24 patients $(24 \%)$ subjected to oral contraceptives therapy or hormone replacement therapy (Table 1). The level of oxidative stress in plasma samples [reactive oxygen metabolites test $\left(\mathrm{d}-\mathrm{ROMs}\right.$ test $\left.\left.\mathrm{t}^{30}\right)\right]$ and antioxidant status in plasma and saliva samples [plasma antioxidant test and saliva antioxidant test (PAT ${ }^{31}$ and $\mathrm{SAT}^{32}$ tests, respectively)], were performed with an easy and quick Point of Care Instrument ( $5 \mathrm{~min}$ for $\mathrm{d}$-ROMs test and 1 min for PAT and SAT tests), by means of FRAS 4 Evolvo (H\&D, Parma, Italy), a photometer and a minicentrifuge kit. Capillary blood samples $(0.15 \mathrm{~mL})$ were collected, though a finger puncture, in a heparinized microcuvette and immediately centrifuged for d-ROMs and PAT tests. In the SAT test, the sampling is prepared chewing for $1 \mathrm{~min}$ a cotton square weighing $300 \mathrm{mg}$ and then squeezing the saliva into a small plastic container.

d-ROMs test: the test is based on the principle that the organic hydroperoxides present in the small amount of plasma $(10 \mu \mathrm{L})$ isolated by centrifugation at $37^{\circ} \mathrm{C}$ for 90 sec, is proportional to free radicals from which they are formed. When the plasma amount is dissolved in an acidic buffer, the hydroperoxides react with the transition metal

\begin{tabular}{lc} 
Table 1: Comparison of risk factors in the \\
enrolled patients \\
\hline Risk factor & Patients (\%) \\
\hline Gender & \\
Male & $100(50)$ \\
Female & $100(50)$ \\
Age group & \\
$<20$ yrs old & $10(5)$ \\
$21-30$ yrs old & $17(9)$ \\
$31-50$ yrs old & $76(38)$ \\
$>50$ yrs old & $97(49)$ \\
Smoking habit & \\
Intense smokers & $46(23)$ \\
Moderate smokers & $27(14)$ \\
Non-smokers & $127(64)$ \\
Diet & \\
Unbalanced diet & $120(60)$ \\
Balanced diet & $80(40)$ \\
Inflammation in the oral cavity & \\
Inflammation & $113(56.5)$ \\
No-inflammation & $87(43.5)$ \\
Hormonal treatment & \\
Hormonal treatment & $24(24)$ \\
No-hormonal treatment & $76(76)$ \\
\hline
\end{tabular}


ions liberated from the proteins in the acidic medium and are converted to alkoxy and peroxy radicals, that oxidize an additive, not coloured chromogen (N,N-diethyl-paraphenylenediamine) to the corresponding radical cation (pink colour). The concentration of these radical species are directly determined through photomer (absorption at $505 \mathrm{~nm}$, Temperature $37^{\circ} \mathrm{C}$ ).

The d-ROMs values are expressed in Units Carratelli (U Carr), whereas $1 \mathrm{U}$ Carr corresponds to $0.08 \mathrm{mg}$ of $\mathrm{H}_{2} \mathrm{O}_{2} / \mathrm{dL}$ and the normal values range between 250 and 300 U Carr (Table 2).

PAT test: it measures the plasma antioxidant status in the presence of zirconium salts added to the reaction mixture: $40 \mu \mathrm{L}$ of $\mathrm{R} 2$ reagent (iron solution) were added to the microcuvette containing R1 reagent (thiocyanate derivate pre-dosed solution), followed by $10 \mu \mathrm{l}$ of the capillary blood sample. The reading was taken at 505 $\mathrm{nm}$ following a 1 -minute incubation period at $37^{\circ} \mathrm{C}$ (temperature of the reading chamber of the photometer). The reference values of PAT test are reported in Table 3 and expressed in U Cor $(1 \mathrm{U}$ Cor $=1.4 \mu \mathrm{mol} / \mathrm{L}$ of ascorbic acid).

SAT test: it measures the saliva antioxidant status in the presence of zirconium salts [salts that bind phosphates (antioxidant competitors) derived from dental demineralization/remineralization processes and present in high quantities in saliva ( $>50 \mathrm{mg} / \mathrm{dL})$ ] added to the reaction mixture: $40 \mu \mathrm{L}$ of $\mathrm{R} 2$ reagent were added to the microcuvette containing R1 reagent, followed by $10 \mu \mathrm{L}$ of the saliva sample. Results were detected with a photometer at $505 \mathrm{~nm}$ following a 1 -minute incubation period at $37^{\circ} \mathrm{C}$. The principle of SAT test is based on the capacity of a iron solution [solution of ferric ions $\left(\mathrm{Fe}^{3+}\right)$ bound to a specific chromogen] to discolour when the $\mathrm{Fe}^{3+}$ ions are reduced to ferrous ions $\left(\mathrm{Fe}^{2+}\right)$ in the presence of phosphates. The reduced ferrous ions are thus quantified, expressing the reducing antioxidant status of the saliva in $\mu \mathrm{mol} / \mathrm{L}$ of Vitamin C (reference standard used as ferrous-reducing agent) (Table 4).

\section{STATISTICAL ANALYSIS}

The statistical comparison of d-ROMs, PAT, and SAT parameters was evaluated using Mann-Whitney test (continuous variables not normally distributed) and chi squared test (categorical variables). A commonly-used measure of linear correlation, the Pearson correlation coefficient, denoted by r, was reported. Statistical significance was set at $p$-value less than 0.05 , and all data and graphics were analyzed using the $\mathrm{R}$ software, version 3.1.2 [R Core Team, Austria (2015)]. ${ }^{33}$

\begin{tabular}{ll} 
Table 2: Reference value of d-ROMs test \\
\hline d-ROMs values (U Carr) & Oxidative stress (OS) severity \\
\hline $250-300$ & Normal value \\
$301-320$ & Borderline value \\
$321-340$ & Slight OS \\
$341-400$ & OS \\
$401-500$ & High OS \\
$>500$ & Very high OS \\
\hline
\end{tabular}

Table 3: Reference value of PAT test

\begin{tabular}{ll}
\hline PAT values (U Cor) & Antioxidant status (AS) \\
\hline$<1800$ & Deficiency status \\
$1801-2000$ & Slight deficiency status \\
$2001-2200$ & Bordeline value \\
$2201-2800$ & Normal value \\
$>2800$ & Very high value \\
\hline
\end{tabular}

Table 4: Reference value of SAT test

\begin{tabular}{ll}
\hline SAT values $(\boldsymbol{\mu m o l} / \mathbf{L}$ Vit $\mathbf{C})$ & Antioxidant status $(\mathbf{A S})$ \\
\hline$<1000$ & Severe shortage \\
$1001-1500$ & Optimal normal value \\
$1501-2000$ & Normal value \\
$2001-2500$ & Borderline value \\
$>2500$ & Possible inflammatory \\
& processes \\
\hline
\end{tabular}

\section{RESULTS}

The mean age of patients was $18 \pm 51.3$ years. There was a slight prevalence of patients with unbalanced diet $(60 \%)$ and inflammatory process in the oral cavity $(56.5 \%)$. For other variables (smoking habit and hormonal treatment) the population was statistically similar $(\mathrm{p}<0.1)$.

\section{d-ROMs, PAT, and SAT tests and gender}

By comparing the d-ROMs test results, the males patients showed a slight oxidative stress ( $300 \pm 340 \mathrm{U}$ Carr), while the female patients showed a significant oxidative stress level $(\mathrm{p}<0.01)$ (Figure 1).

As to antioxidant status (PAT test) little significant differences were observed between genders $(\mathrm{p}=0.054)$ : a slightly higher value $(27 \%)$ was detected in women compared to men (12\%).

The median SAT value was $1625 \mu \mathrm{mol} / \mathrm{L}$ Vit C [IQR (11802390)] for the male group and $1627 \mu \mathrm{mol} / \mathrm{L}$ Vit $\mathrm{C}[\mathrm{IQR}$ (1193-2176.5)] for the female group. The statistical analysis of this test showed no significant sex difference $(\mathrm{p}=0.582$ ).

\section{d-ROMs, PAT and SAT tests and age}

We analyzed the oxidative stress and antioxidant status in relation to age, dividing the study population in two age groups ( $\leq 50$ years and $>50$ years) and we registered higher and little significant d-ROMs values and lower, but no 
significant, PAT values, in ageing patients $(\mathrm{p}=0.054$ and 0.488 , respectively).

As shown in Figure 2, there is a marked and significant increase of SAT values with the increasing of age $(\mathrm{p}<0.01)$.

\section{d-ROMs, PAT and SAT tests and smoking habit}

The d-ROMs values in plasma of intense smokers were increased, but non significantly, when compared to nonsmokers and moderate smokers. Comparing the data obtained for the three groups (non-, moderate and intense smokers) no significant difference between oxidative stress (d-ROMs test) and plasma antioxidant status (PAT test) was found ( $p=0.24$ and 0.64 , respectively).

In the intense smoking group it was stated that SAT values in the saliva samples analyzed have normal values: $1641 \mu \mathrm{mol} / \mathrm{L}$ Vit C [IQR (1094-2363)]. However, we also registered higher SAT values in the non-smokers but with inflammation of the oral cavity $\{2027 \mu \mathrm{mol} / \mathrm{L}$ Vit C IIQR (753-7488)\}, compared to non- and moderate smoking

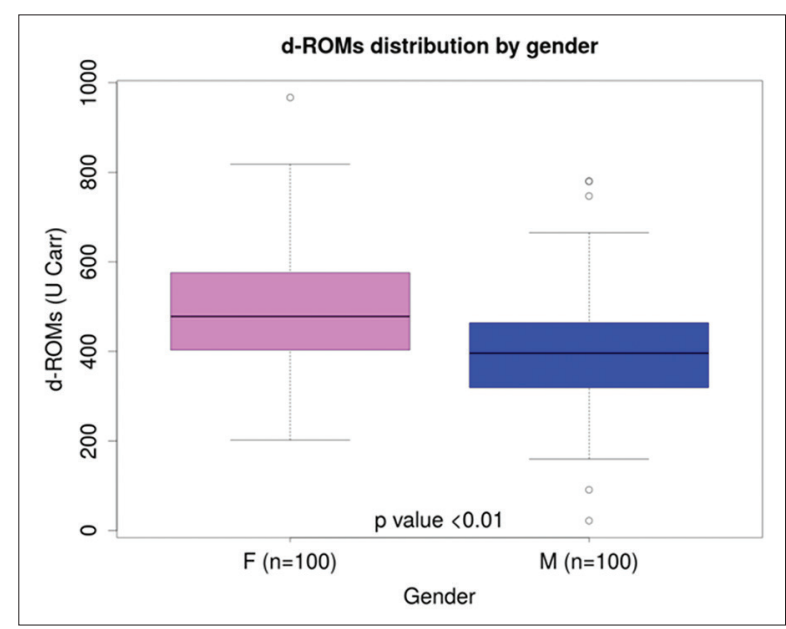

Figure 1: Relationship d-ROMs values-gender

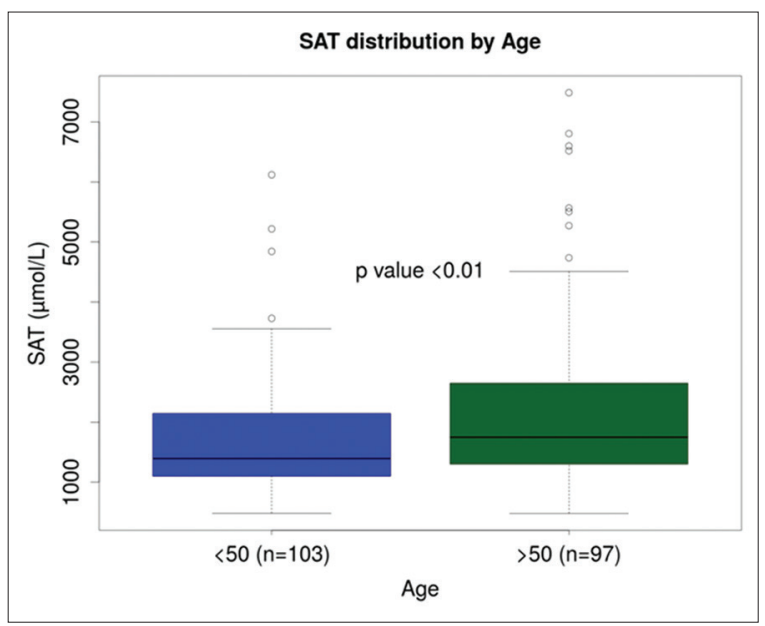

Figure 2: Relationship SAT values-age groups, that should it be adaptive response to the increase of free radicals associated to the inflammation.

d-ROMs, PAT and SAT tests and unbalanced diet As shown in Figures 3 and 4, high, but no significantly, $\mathrm{d}$-ROMs values and significant decrease of PAT levels in patients with unbalanced diet were observed $(\mathrm{p}=0.40$ and $\mathrm{p}<0.01$, respectively).

Specifically, higher d-ROMs levels were detected in women with unbalanced diet $\{490 \mathrm{U}$ Carr [IQR (216-967)] $\}$, compared to men. Antioxidant influence in the saliva samples, evaluated with SAT test, remained unchanged in the both groups: $1578 \mu \mathrm{mol} / \mathrm{L}$ Vit C [IQR (1178-2274)] and $1688 \mu \mathrm{mol} / \mathrm{L}$ Vit C [IQR (1200-2348)] in balanced and unbalanced diet.

d-ROMs, PAT and SAT tests and inflammation in the oral cavity

d-ROMs test showed, statistically, a significant increase $(\mathrm{p}=0.04)$ in the patients with inflammatory process in

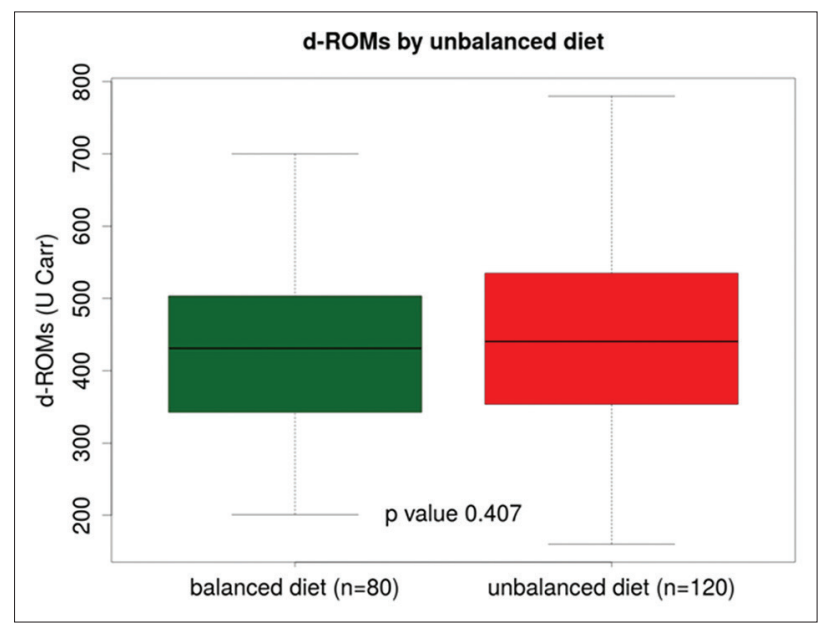

Figure 3: Relationship d-ROMs values-unbalanced diet

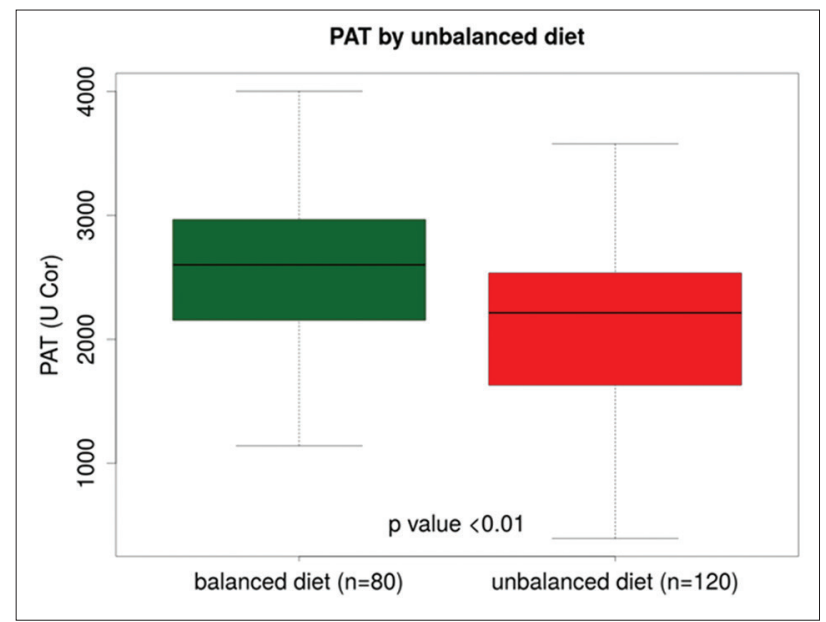

Figure 4: Relationship PAT values-unbalanced diet 
the oral cavity compared to values obtained in the other patients (Figure 5).

No significant PAT values in both groups (healthy patients and patients with inflammatory condition) were observed.

We evidenced higher and significant SAT values $(\geq 3000 \mu \mathrm{mol} / \mathrm{L})$ in the subjects with inflammatory condition, specifically in the non-smoking group with oral inflammation $\{2027 \mu \mathrm{mol} / \mathrm{L}$ Vit C [IQR $(753$ $7488)\}$, indicating the need to the further evaluation and identification of the pathological cause (Figure 6).

d-ROMs, PAT, and SAT tests and hormonal treatment The differences of oxidative/antioxidant status in female gender would be due to the hormonal status (e.g. oral contraceptives or hormone replacement therapy). We compared the female samples divided into two groups: non-administration $(n=76)$ and administration $(n=24)$ of hormonal treatment.

Comparing these two groups a difference, but not significant, was observed in the values of oxidative stress (d-ROMs test) (Figure 7). Indeed, the d-ROMs values of the second group (hormonal treatment) are in the range of very high oxidative stress, $474 \mathrm{U}$ Carr [IQR $(415,629)]$; but the same group showed normal values of antioxidant status (PAT test): 2596 U Cor [IQR $(2374,2989)]$, which could promote a balance between free radicals and antioxidants in these patients.

We also registered high d-ROMs levels in postmenopausal women (over 50 yrs) but not using hormonal therapy $\{510$ U Carr [QR (247-755)] \} accordingly with the values observed in other variables (e.g. age).

There were no significant differences in SAT levels between women undergoing hormonal treatment and those not treated: $1393 \mu \mathrm{mol} / \mathrm{L}$ Vit C [IQR (1204,2096)] vs 1648 $\mu \mathrm{mol} / \mathrm{L}$ Vit C [IQR $(1184,2186)]$.

\section{DISCUSSION}

The assessment of oxidative stress and plasma/salivary antioxidant status represents a measure of physiological, environmental, and nutritional factors (including exposure to ROS and antioxidant supplementation), and allows to correlate the main health risk factors to oxidative stress, involved in the development/progression of disease.

\section{d-ROIIs, PAT, and SAT tests and gender}

The oxidative stress was greater in women than in men at the same age: the female group, specifically postmenopausal women (over $50 \mathrm{yrs}$ ) with unbalanced

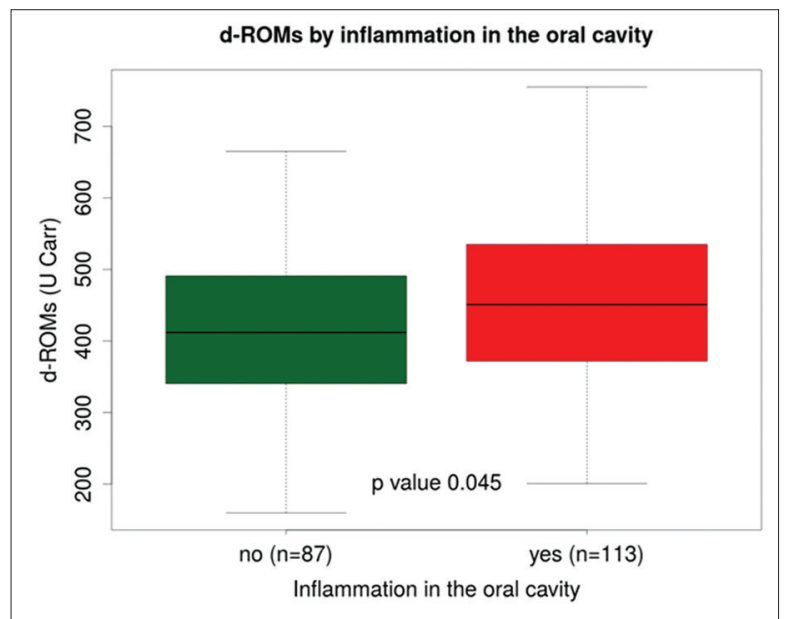

Figure 5: Relationship d-ROMs values-inflammation in oral cavity

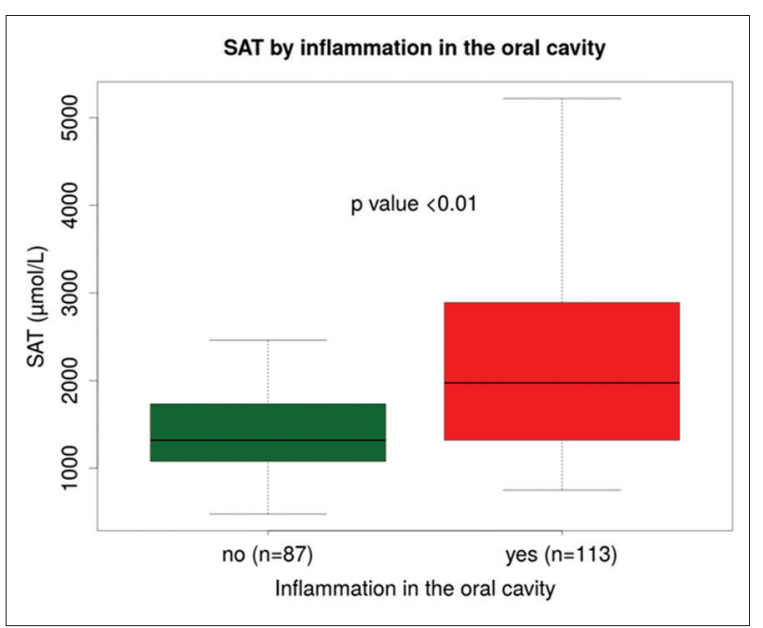

Figure 6: Relationship SAT values-inflammation in oral cavity

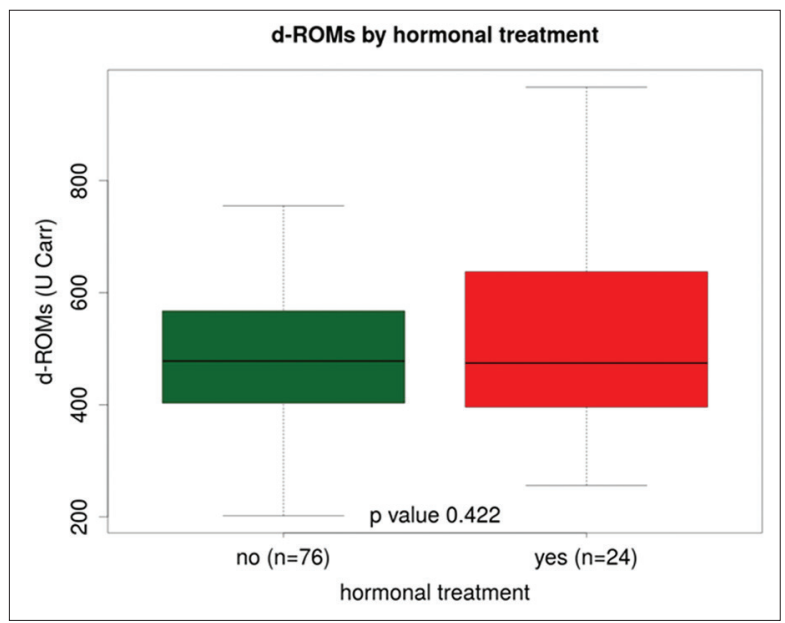

Figure 7: Relationship d-ROMs values-hormonal treatment

diet and not administered to hormonal therapy, showed an high level of d-ROMs in blood samples with normal antioxidant status of the blood and saliva samples (PAT and SAT tests). Female have stronger cellular and 
humoral immune reactions compared with men and sex hormones (e.g. estrogen) can be partly responsible for the higher occurrence of autoimmune disorders in these patients. ${ }^{34}$ Indeed, the estrogens have immune modulating properties, influence the innate and adaptive immune cells, antigen presentation, cytokine secretion, and generation of autoantibodies and stimulate autoreactive B-cells, promoting the escape of autoreactive cells from the mechanisms of immune tolerance. ${ }^{8}$

A link between cardiovascular disease and lung cancer in women, through oxidative stress has been supposed by a study run on a large cohort of patients with previous atherosclerotic disease-the female patients are more sensitive to tobacco-induced carcinogenesis than men, since a higher level of lung cytochrome P4501A1 (CYP1A1) gene expression in women plays a significant role in lung DNA adduct formation and supports a higher susceptibility to lung cancer among women. ${ }^{35}$

Furthermore, we can suppose that the gender-different antioxidant status added to the less frequent women daily physical exercise, and/or the combination with other risk factors including ageing, smoking habit, obesity might share a co-aetiologic role in the diseases, such as cardiovascular diseases.

\section{d-ROMs, PAT and SAT tests and age}

The high d-ROMs values detected in two age groups $(\leq 50$ and $>50)$ are in agreement with our previously described data which show a higher level of oxidative stress in postmenopausal women (over $50 \mathrm{yrs}$ ).

These values attended by lower PAT values play a key role in the molecular mechanism of specific age-related eye diseases such as nuclear cataract: an opaqueness of crystalline lens confined to the true nucleus of the lens, whose the shape is determined by the concentric arrangement of the fibers that compose the lens. ${ }^{36}$ In lens tissue, excessive amounts of ROS determine degradation, cross-linking, and aggregation of lens proteins; which lead to lens opacity and the augmented accumulation of fluorescent chromophores. ${ }^{37}$ The other risk factor associated to age-related cataract is smoking that causes heavy metals accumulation (cadmium, lead, and copper) in the lens or increase of isocyanates and aldehydes levels in the blood of smokers, that can modify lens proteins, causing lens opacification. ${ }^{38}$

A significant age-related increase of SAT levels might be induced by age-related changes in the salivary gland parenchyma and/or by worsening of the mouth hygienic or functional conditions [dental infections (e.g. caries, periodontitis), tooth loss, benign mucosal lesions, and oral cancer], and the increased oral therapy in elderly persons.
d-ROMs, PAT and SAT tests and smoking habit It is found a higher increase of d-ROMs level, but normal PAT values, in intense smokers group, nevertheless other putative risk co-factors, such as age, gender, inflammation condition and obesity can be responsible of redox imbalance. ${ }^{39}$ The presence of $10^{14}-10^{16}$ oxidants/puff of cigarette increases the production of inflammation mediators, with subsequent obstructive pulmonary disease and lung cancer.

The normal, and non severe shortage, SAT values, in the intense smoking group, might express the endogenous neutralization of the free radicals produced by tobacco smoking; as well as the raised SAT values in the nonsmokers but with chronic inflammation of the oral cavity could represent an adaptative response to the increase of free radicals associated to the inflammation.

\section{d-ROMs, PAT and SAT tests and unbalanced diet}

The higher ROS increase (higher d-ROMs values) and significant decrease of antioxidant status (lower PAT values) registered in the group with high-fat or unbalanced diet, specifically in women, is associated with increased NADPH oxidase concentration and depletion of exogenous antioxidants (inadequate dietary supply, unbalanced/poor nutrition), and it pathway may lead to cell damage and consequently to the disease development. ${ }^{40}$

In the CNS pathology, including Parkinson's disease (PD), Alzheimer's disease (AD), Multiple Sclerosis (MS) and amyolotrophic lateral sclerosis (ALS), the unbalanced oxidative/antioxidant status is supposed to have a key causative role. ${ }^{41}$ When the ROS level exceeds the total detoxifying capacity of the mitochondria, the mitochondrial transmembranes permeability transition pores (mtPTP), are increased, opening channels to solutes diffusion with molecular mass smaller than $1.5 \mathrm{kDa}$ between the matrix and the cytosol. ${ }^{42}$ This determines the trans-membrane electrochemical gradient collapse, with loss of matrix solutes, mitochondrial wrecking, and release of cytochrome $c$, procapases 2, 3, and 9, apoptosis-inducing factor (AIF), and caspase activated DNase (CAD). Cytochrome $c$ and the cytosolic apoptotic protease activating factor 1 (Apaf1) activate the caspases, while AIF and CAD move to the nucleus initiating apoptosis or programmed cell death. ${ }^{43}$

A longitudinal study of 4606 elderly individuals without dementia but with unbalanced diet showed that higher intake of total calories and fats is associated with higher risk of $\mathrm{AD}$ in carriers of the apolipoprotein $\mathrm{E}$ (APOE) e4 allele, supporting the hypothesis that caloric restriction, intake of n-3 PUFAs as well as DHA, may reduce the development of neurodegenerative diseases. ${ }^{44}$ 
d-ROMs, PAT and SAT tests and inflammation in the oral cavity

When the oxygen free radicals increases, the saliva [a mixture of major and minor salivary gland secretions, gingival crevicular (sulcular) fluid, bronchial and nasal secretions, serum and blood derivatives from oral wounds, microorganisms, leukocytes, desquamated epithelial cells, and food debris in patients with oral cavity inflammation (e.g. bleeding gums, dental caries)] increases the antioxidant level as a compensatory oxidative stress counteracting reaction. ${ }^{45}$

The enhanced salivary antioxidant status (SAT test) can be induced by localized oral inflammation but also by systemic oxidative background, and it could be linear with plasma antioxidant response (PAT test).

Indeed, the higher and significant d-ROMs and SAT values registered in the patients with inflammation condition are directly proportional to the severity of oral inflammation and can be useful markers in healthy people but also in fluctuation of diabetes and cancer (e.g. oral cancer) progression, because the latters have increased salivary antioxidant parameters, cytokines such as IL-8, IL-6, and growth factors such as epidermal growth factor (EGF). ${ }^{46-48}$ Astaneie and coworkers ${ }^{49}$ evaluated the total serum and salivary antioxidant status of 19 patients with type 1 diabetes mellitus and 19 healthy patients and found that salivary antioxidant capacity of diabetic patients was higher $(p<0.01)$ than that of the control group. These patients showed an increased level of EGF, a growth factor secreted from salivary glands (measured by ELISA), in salivary sample, but reduced level in plasmatic sample. It is supposed that this difference between saliva and blood could be due to the impaired absorption of EGF through the gastrointestinal tract in diabetic patients: nitrergic neurons, innervating the urogenital and gastrointestinal organs undergo a specific degenerative process that determines an initial insulin-reversible decrease in neuronal NO synthase (nNOS) in the axons, followed by apoptosis of the nitrergic neurones, a process that is not reversible by insulin. ${ }^{50}$ Consequently, salivary glands are stimulated to secrete EGF to help beta cells to secrete insulin.

Similarly, a case-control study conducted on 22 oral squamous cell carcinoma patients (about the $40 \%$ of patients had a history of tobacco smoking) showed significant increase of salivary antioxidant level compared with 22 healthy controls $(p=0.029) .{ }^{45}$

d-ROMs, PAT, and SAT tests and hormonal treatment Our data confirmed that the hormonal treatment, including administration of oral contraceptives or hormone replacement therapy, increases the oxidative stress in the women, as evaluated by d-ROMs test. One limit of this study was the non assessment of the specific phase of the menstrual cycle, because the women are in oxidative stress condition for a central phases of the cycle (late follicular phase and early luteal phase) or rather at the time of ovular maturation and possible implantation: the oxidative stress peak corresponds to their estrogen peak, and tends to decrease slightly when progestin hormone increases. ${ }^{51}$

We observed no effect of the hormonal treatment on the plasma and saliva antioxidant status (PAT and SAT tests), but only a possible relationship between the postmenopausal women (over 50yrs) and no hormonal therapy, and the cumulative effect of tobacco smoking (another factor of cardiovascular risk) and hormonal treatment, accordingly with other authors. ${ }^{52}$

\section{CONCLUSIONS}

The present findings provide additional information regarding oxidative stress-related health risk factors in healthy patients and suggest the use of a non-invasive, simple, precise, reliable and fast Point of Care Tests (POCTs), in clinical practice, on the prevention diagnosis, prognosis, and treatment effectiveness in some specific diseases.

Furthermore, salivary antioxidant status evaluation achieved with an easy, not invasive and repeatable procedure, and inflammation in the oral cavity are obviously related. Unlike blood, which is obtained invasively, saliva can be easily collected and the rapid SAT test could be a good alternative for blood in diagnosis or screening of oral \& periodontal disease but also type 1 diabetes and oral cancer.

In conclusion, this study was intended to define the baseline of redox status on an healthy recruited population, as the very preliminary step to assess the antioxidant/oxidant progressive diseases inducing role of some very common illnesses including cardiovascular diseases, fibromyalgia and cancer. As to the high levels SAT detected in otherwise healthy people they are not related to the measure of systemic oxidative stress and antioxidant response, but rather to chronic mouth (dental-parodontal problems) and should be taken in account to prevent leukoplakia and oral cancer on a multifactorial basis.

\section{ACKNOWLEDGEMENTS}

The authors thank the Company H\&D SRL (Parma, Italy) for the Point of Care and reagents. 


\section{REFERENCES}

1. Halliwell B. Oral inflammation and reactive species: a missed opportunity? Journal of Oral Diseases 2000; 6(3):136-137.

2. Urtasun R, De la Rosa LC and Nieto N. Oxidative and nitrosative stress and fibrogenic response. Clinical Liver Disease 2008; 12(4):769-790.

3. Murphy E. Estrogen signaling and cardiovascular disease. Circulation Research 2011; 109(6): 687-696.

4. Chapple IL, Mason GI, Garner I, Matthews JB, Thorpe GH, et al. Enhanced chemiluminescent assay for measuring the total antioxidant capacity of serum, saliva and crevicular fluid. Annals of Clinical Biochemistry 1997; 34 (4): 412-421.

5. Vignon-Zellweger N, Relle K, Rahnenfuhrer J, Schwab K, Hocher B and Theuring F. Endothelin-1 overexpression and endothelial nitric oxide synthase knock-out induce different pathological responses in the heart of male and female mice. Life Sciences 2014; 118(2): 219-225.

6. Sumi D and Ignarro LJ. Estrogen-related receptor alpha 1 up-regulates endothelial nitric oxide synthase expression. Proceedings of the National Academy of Sciences 2003; 100(24):14451-14456.

7. Ahtarovski KA, Iversen KK, Christensen TE, Andersson $H$, Grande P and Holmvang L. Termination of dobutamine infusion causes transient rebound left heart diastolic dysfunction in healthy elderly women but not in men: a cardiac magnetic resonance study. American Journal of Physiology Heart Circulatory Physiology 2013; 305(7):1098-1103.

8. Kamhieh-Milz J and Salama A. Oxidative stress is predominant in female but not in male patients with autoimmune thrombocytopenia. Oxidative Medicine and Cellular Longevity 2014; 1-9.

9. Kurien BT and Scofield RH. Autoimmunity and oxidatively modified autoantigens. Autoimmunity Reviews 2008;7(7): 567573.

10. Romano AD, Serviddio G, de Matthaeis A, Bellanti F and Vendemiale G. Oxidative stress and aging. Journal of Nephrology 2010; 23 (15): S29-S36.

11. Junqueira VB, Barros SB, Chan SS, Rodriques L, Giavarotti L, Abud RL, et al. Aging and oxidative stress. Molecular Aspects of Medicine 2004; 25(2): 5-16.

12. Rahman I and MacNee W. Role of oxidants/antioxidants in smoking-induced lung diseases. Free Radical Biology \& Medicine 1996; 21(5): 669-681.

13. Smith $\mathrm{CJ}$ and Hansch $\mathrm{C}$. The relative toxicity of compounds in mainstream cigarette smoke condensate. Food and Chemical Toxicology 2000; 38(7): 637-646.

14. Lykkesfeldt J, Christen S, Wallock LM, Chang HH, Jacob RA and Ames BN. Ascorbate is depleted by smoking and repleted by moderate supplementation: a study in male smokers and nonsmokers with matched dietary antioxidant intakes. American Journal Clinical Nutrition 2000; 71(2):530-536.

15. Leitzmann C. Adequate diet of essential nutrients for healthy people. The role of food, agriculture, forestry and fisheries in human nutrition. Encyclopedia of Life support systems 2009; 4:1-9.

16. Lugrin J, Rosenblatt-Velin N, Paranov R and Liaudet L. The role of oxidative stress during inflammatory processes. Biological Chemistry 2014; 395(2):203-230.

17. Chan JK, Roth J, Oppenheim JJ, Tracey KJ, Vogl T, Feldmann $M$, et al. Alarmins: awaiting a clinical response. Journal of Clinical Investigation 2012; 122(8): 2711-2719.

18. Charlton BM, Rich-Edwards JW, Colditz GA, Missmer SA, Rosner BA, Hankinson SE, et al. Oral contraceptive use and mortality after 36 years of follow-up in the Nurses' Health Study: prospective cohort study. British Medical Journal 2014; 349:6356-6362.

19. Spencer AL, Bonnema R and McNamara MC. Helping women choose appropriate hormonal contraception: update on risks, benefits, and indications. American Journal of Medicine 2009; 122(6): 497-506.

20. Collins P, Rosano GM, Sarrel PM, Ulrich L, Adamopoulos S, Beale CM, et al. 17 beta-Estradiol attenuates acetylcholineinduced coronary arterial constriction in women but not men with coronary heart disease. Circulation 1995; 92(1): 24-30.

21. Kose K, Dogan P and Ozesmi C. Contraceptive steroids increase erythrocyte lipid peroxidation in female rats. Contraception 1993; 47(4): 421-425.

22. Sissan MA, Menon VP and Leelamma S. Effects of low-dose oral contraceptive oestrogen and progestin on lipid peroxidation in rats. Journal of International Medical Research 1995; 23(4): 272-278.

23. Ciavatti M, Blache D and Renaud S. Hormonal contraceptive increases plasma lipid peroxides in female rats. Relationship to platelet aggregation and lipid biosynthesis. Arteriosclerosis 1989; 9(1): 84-89.

24. Ciavatti M and Renaud S. Oxidative status and oral contraceptive. Its relevance to platelet abnormalities and cardiovascular risk. Free Radical Biology \& Medicine 1991; 10(5): 325-338.

25. Horwitt MK, Harvey $\mathrm{CC}$ and Dahm $\mathrm{CH}$. Relationship between levels of blood lipids, vitamins C, A, and E, serum copper compounds, and urinary excretions of tryptophan metabolites in women taking oral contraceptive therapy. American Journal of Clinical Nutrition 1975; 28(4): 403-412.

26. Pasqualini JR. Differential effects of progestins on breast tissue enzymes. Maturitas 2003; 46 (1): S45-S54.

27. Wunsch A and Palmieri B. The role of second opinion in oncology: an update. European Journal of Oncology 2013; 18(3): 1-5.

28. Palmieri B, lannitti $\mathrm{T}$, Capone $\mathrm{S}$ and Arisi E. [Second opinion clinic: is the Web Babel Syndrome treatable?]. Clinica Tereapeutica 2011; 162(6):575-583.

29. Palmieri B and lannitti T. The Web Babel syndrome. Patient Education and Counseling 2011; 85(2):331-333.

30. Cornelli U, Terranova R, Luca S, Cornelli M and Alberti A. Bioavailability and antioxidant activity of some food supplements in men and women using the D-Roms test as a marker of oxidative stress. Journal of Nutrition 2001; 131(12): 3208-3211.

31. Benedetti S, Primiterra M, Catalani S, Finco A, Canestrari F, et al. Performance evaluation of the innovative PAT test, comparison with the common BAP test and influence of interferences on the evaluation of the plasma antioxidant capacity. Clinical Laboratory 2013; 59(10):1091-1097.

32. Benedetti S, Primiterra M, Finco A and Cornelli $U$. Validation of a patented method to determine the antioxidant capacity of human saliva based on the reduction of iron: the SAT test. Clinical Laboratory 2014; 60(3):475-482.

33. R Development Core Team. A Language and Environment for Statistical Computing. R Foundation for Statistical Computing, Vienna, Austria 2015. URL https://www.R-project.org/.

34. Invernizzi P, Pasini S, Selmi C, Gershwin ME and Podda M. Female predominance and Xchromosome defects in autoimmune diseases. Journal of Autoimmunity 2009; 33(1):12-16.

35. Mollerup S, Berge G, Baera R, Skaug V, Hewer A, Phillips DH, et al. Sex differences in risk of lung cancer: Expression of genes in the PAH bioactivation pathway in relation to smoking and bulky DNA adducts. International Journal of Cancer 2006. 119(4):741-744.

36. Michael R and Bron AJ. The ageing lens and cataract: a model 
of normal and pathological ageing. Philosophical Transactions of the Royal Society of London B Biological Sciences 2011; 366(1568): 1278-1292.

37. Hanson SR, Hasan A, Smith DL and Smith JB. The major in vivo modifications of the human water-insoluble lens crystallins are disulfide bonds, deamidation, methionine oxidation and backbone cleavage. Experimental Eye Research 2000; 71(2):195-207.

38. Racz $P$ and Erdohelyi A. Cadmium, lead and copper concentrations in normal and senile cataractous human lenses. Ophthalmic Research 1988; 20(1):10-13.

39. Jansen EHJM, Beekhof $P$ and Ruskovska T. The Effect of Smoking on Biomarkers of (Anti) oxidant Status. Journal of Molecular Biomarkers \& Diagnosis 2014; 5(6): 1-4.

40. Avelar TMT, Storch AS, Castro LA, Azevedo GVMM, Ferraz L, et al. Oxidative stress in the pathophysiology of metabolic syndrome: which mechanisms are involved? Journal Brasileiro de Patologia e Medicina Laboratorial 2015; 51(4): 231-239.

41. Uttara B, Ajay $V$ and Mahajan RT. Oxidative stress and neurodegenerative diseases: a review of upstream and downstream antioxidant therapeutic options. Current Neuropharmacology 2009; 7(1): 65-74.

42. Emerit J, Edeas $\mathrm{M}$ and Bricaire F. Neurodegenerative diseases and oxidative stress. Biomedicine Pharmacotherapy 2004; 58(1): 39-46.

43. Kokoszka JE, Coskun P, Esposito LA and Wallace DC. Increased mitochondrial oxidative stress in the Sod2 (+/-) mouse results in the age-related decline of mitochondrial function culminating in increased apoptosis. Proceedings of the National Academy Sciences of the United States of America 2001; 98(5): 22782283.

44. Luchsinger JA, Tang MX, Shea S and Mayeux R. Caloric intake and the risk of Alzheimer disease. Archives of Neurology 2002; 59(8):1258-1263.

45. Najafi $\mathrm{SH}$, Gholizadeh $\mathrm{N}$, Manifar $\mathrm{S}$, Rajabzadeh $\mathrm{S}$ and Kharazi FMJ. Salivary Antioxidant Level in Oral Squamous Cell Carcinoma. Iranian Journal of Blood \& Cancer 2015; 7(2): 57-60.

46. Osman TA, Costea DE and Johannessen AC. The use of salivary cytokines as a screening tool for oral squamous cell carcinoma: A review of the literature. Journal of Oral Maxillofacial Pathology 2012; 16(2):256-261.

47. Nunes LA, Mussavira $S$ and Bindhu OS. Clinical and diagnostic utility of saliva as a non-invasive diagnostic fluid: a systematic review. Biochemia Medica (Zagreb) 2015; 25(2):177-192.

48. Brano S, David MP and Indira AP. Salivary Biomarkers for Oral Squamous Cell Carcinoma: An Overview. International Journal of Scientific Studies 2015; 1(8): 39-45.

49. Astaneie F, Afshari M, Mojtahedi A, Mostafalou S, Zamani MJ, Larijani B, et al. Total antioxidant capacity and levels of epidermal growth factor and nitric oxide in blood and saliva of insulindependent diabetic patients. Archivies Medicine Research 2005; 36(4): 376-381.

50. Cellek S, Qu W, Schmidt AM and Moncada S. Synergistic action of advanced glycation end products and endogenous nitric oxide leads to neuronal apoptosis in vitro: a new insight into selective nitrergic neuropathy in diabetes. Diabetologia 2003; 47 (2): 331-339.

51. Cornelli, $U$, Belcaro $G$ and Finco A. Analysis of oxidative stress during the menstrual cycle. Reproductive Biology Endocrinology 2013; 11: 74-80.

52. Palan, PR, Magneson AT, Castillo M, Dunne $\mathrm{J}$ and Mikhail MS. Effects of menstrual cycle and oral contraceptive use on serum levels of lipid-soluble antioxidants. American Journal of Obstetrics and Gynecology 2006; 194(5): e35-e38.

Authors Contribution:

CL - Manuscript Preparation, Editing; BP- Manuscript Preparation, Editing; AM - Data acquisition and Statistical analysis; MV - Manuscript Preparation and Editing.

Source of Support: Nil, Conflict of Interest: None declared. 